\title{
A mass estimate for a sample of nearby stars
}

\author{
Slobodan Ninković and Veselka Trajkovska \\ Astronomical Observatory, Volgina 7, 11160 Belgrade 74, Serbia and Montenegro \\ email: sninkovic@aob.bg.ac.yu \\ email: vtrajkovska@aob.bg.ac.yu
}

\begin{abstract}
We form a sample of nearby single stars. Their masses are estimated on the basis of the mass-luminosity relation. Our aim is to specify a reasonable space limit, i.e. a heliocentric volume, as small as possible, but to contain a sufficient number of stars so that an application of statistical laws becomes possible. Among the quantities calculated by us a special attention is paid to the mean mass of a single star and, also, to the local value of Agekyan's factor.
\end{abstract}

Keywords. Stellar mass, nearby stars

\section{Introduction}

It is sufficiently well known that for a star its mass is, practically, its most important physical property, but also that to determine reliably the mass of an individual star is a difficult task. The data sources for stellar masses are binary stars. However, this case is not without difficulties. Even if the question, whether a stellar pair considered as a binary is really bound, is put aside, nevertheless, problems are still present; for some kinds of binaries, say visual, the total mass can be found, whereas in the case of, say spectroscopic binaries, the first quantity to be determined is the mass ratio. Despite of all of this masses of many stars have been determined (better to say, estimated) and, as a consequence, an empirical rule was obtained, according to which for, at least, the stars of the main sequence of the HR diagram the higher the luminosity is, the larger is the mass (for details see Angelov 1993, and the references therein). Such a relationship is to be expected bearing in mind the energy sources of stars.

Once the masses for a sufficiently large number of stars are known, it is possible to analyse the mass distribution of stars. There are mathematical models developed for this purpose (e.g. Marochnik \& Suchkov 1984, p. 200, and the references therein). Among the questions inestigated by one of the present authors (Ninkovic 1995), of importance is the value of Agekyan's factor in stellar systems. In the present paper an attempt is done to answer these questions.

\section{Theory}

Agekyan's factor is a dimensionless quantity appearing in the formula for a criterion aimed at estimating the role of regular, i.e. irregular forces in stellar systems. The criterion was proposed by Agekyan (1962). Such criteria are normally based on a characteristic ratio indicating the importance of irregular, i.e. regular forces. The concept of dividing gravitational forces into these two groups is almost fundamental in stellar dynamics. The regular forces are described by means of the gravitational potential which has its origin in the mass distribution within a given stellar system, whereas the irregular forces have a stochastic nature and they are usually manifested through stellar encounters. Agekyan estimates the volume of the part of a stellar system within which the irregular forces 
prevail over the regular ones. As the unit he uses the total volume of that stellar system. In this way we have the following expression:

$$
A=\frac{2}{\sqrt{N}} \frac{\left\langle m^{3 / 2}\right\rangle}{\langle m\rangle^{3 / 2}}
$$

As easily seen, the first factor on the right hand side of this equation is a simple decreasing function of $\mathrm{N}$ (total number of stars in the stellar system). This is to be expected because the higher the total number of stars, the less is the importance of irregular forces; however the factor depending on star masses is something new, in which Agekyan's criterion is different from other similar criteria, since it indicates that in addition to the total number of stars the mass distribution (mass scatter) is also important in estimating the importance of irregular forces. For this reason this factor is called Agekyan's one, and it is treated in the present paper.

It should be mentioned that the action of irregular forces is also characterised by the amount of the so-called relaxation time (time interval within which the moduli of the average change of mechanical energy per star and of the average mechanical energy per star become equal to each other). The best solution is to express it in terms of the socalled crossing time (time interval needed on the average for a star to cross the system diameter). This ratio also depends on N (e.g. Binney \& Tremaine 1987 - eq. (8.1), p. 489), also being a simple function of it and leading to the same conclusions as the first factor in Agekyan's formula.

\section{Mass Estimates}

The data source is the Hipparcos Catalogue (ESA 1997). It contains the parallaxes, apparent magnitudes and other data for a large number of stars (more than 100000 ). We expect these parallaxes to be accurate enough so that they enable deriving of absolute magnitudes for all stars with known apparent magnitudes. Since the Hipparcos stars are sufficiently close to the Sun, the interstellar absorption is practically negligible. As the relation yielding the mass of a star on the basis of its luminosity, we use the one proposed by Angelov 1993. The input data for this relation are visual absolute magnitudes and, consequently, there is no need to calculate the corresponding bolometric magnitudes. We form the input parameters (star magnitudes, in fact the apparent magnitudes corrected for the distance, i.e. parallax), and as the output we have the masses. Our previous experience (Trajkovska \& Ninković 1997) shows that the star masses determined in this way can be reliable enough. For this reason we insist on single stars only because the astrophysical data necessary for our estimation are more reliable in the case of single stars.

\section{Results}

Our first step is to separate those Hipparcos stars with parallaxes exceeding 0".01. Of course, of interest are only the cases where all the data necessary for our work are available. In this set of stars we look for the ones with designation $\mathrm{V}$ in the Catalogue which indicates the main sequence (MS), because the relation for mass estimation used by us is valid for MS only. The corresponding HR diagram is presented in Fig. 1.

Within this sample (Sample 1) we form a subsample containing very close stars (Sample 2), with parallaxes greater than 0".1. The sample containing stars with parallaxes between 0".01 and 0".1 is referred to as Sample 3. The parallax distribution is presented 


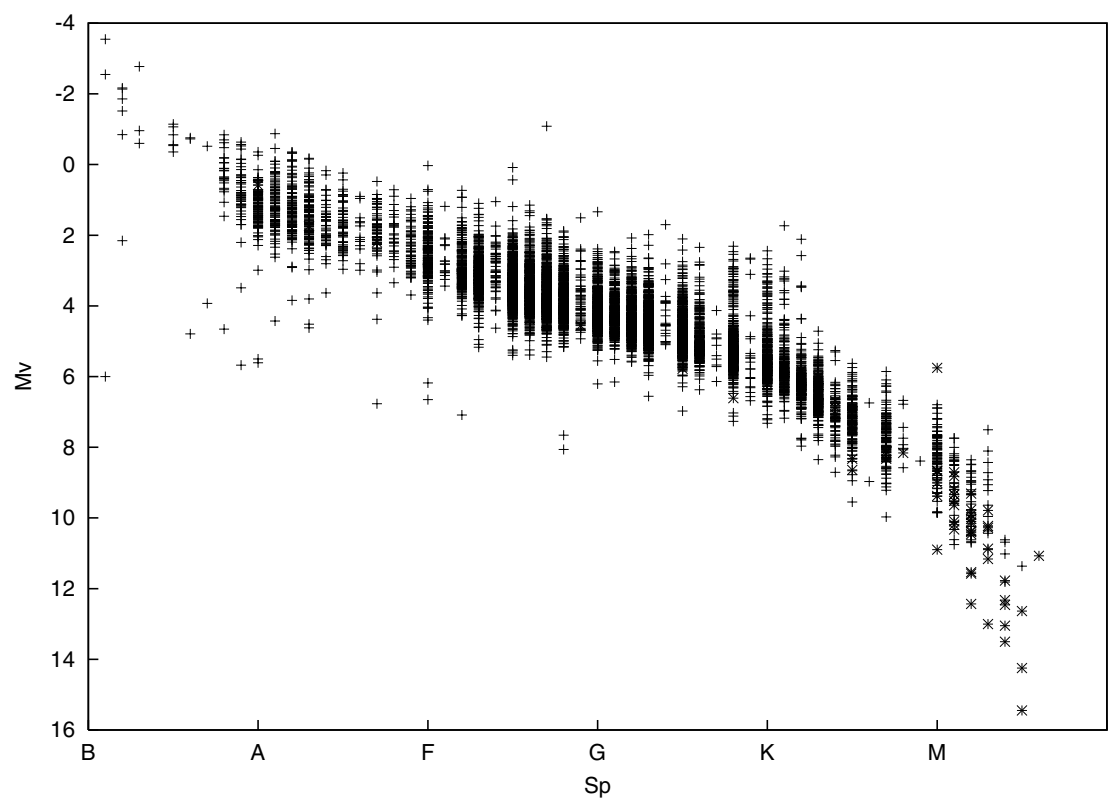

Figure 1. HR Diagram for Stars of Sample 1.

Table 1. The mean mass of a single star, standard deviation and the value of Agekyan's factor for all samples.

\begin{tabular}{lrccc}
\hline Sample & $n$ & $\langle m\rangle$ & $\sigma_{\langle m\rangle}$ & $A$ \\
\hline Sample 1 & 9496 & 1.288 & 0.582 & 1.071 \\
Sample 2 & 96 & 0.602 & 0.495 & 1.221 \\
Sample 3 & 9400 & 1.295 & 0.579 & 1.069 \\
\hline
\end{tabular}

in Fig. 2 for Sample 1 (above), and for Sample 2 (below). As for the parallax distribution for Sample 3, it is very similar to that for Sample 1. The reason is very simple, Sample 2 contains a very small number of stars as will be shown below. Thus, separate plots for Sample 3 are not given.

For each star we calculate the mass by applying Angelov's (1993) relation. The mass distribution is presented in Fig. 3 for Sample 1 (above), and for Sample 2 (below).

For all these samples we calculate the mean mass of a single star, its standard deviation and the value of Agekyan's factor. These values are given in Table 1. The number of stars in each sample is given in the first column. Note that the number of stars within a sample is in no way connected with the total number of stars in a stellar system $(\mathrm{N}$ in the formula of Agekyan's criterion). Here the stellar system under study is the Galaxy, thus N would be of order of $10^{11}$. The unit for the mean mass and its standard deviation is the mass of the Sun.

\section{Discussion and Conclusion}

First, it should be emphasized that in the case of Sample 2 the mean mass of a single star is significantly different from the values obtained for the other two samples which are very close to each other. The same can be said for the case of Agekyan's factor, a dimensionless quantity characterising the scatter in the star masses. As for the standard 

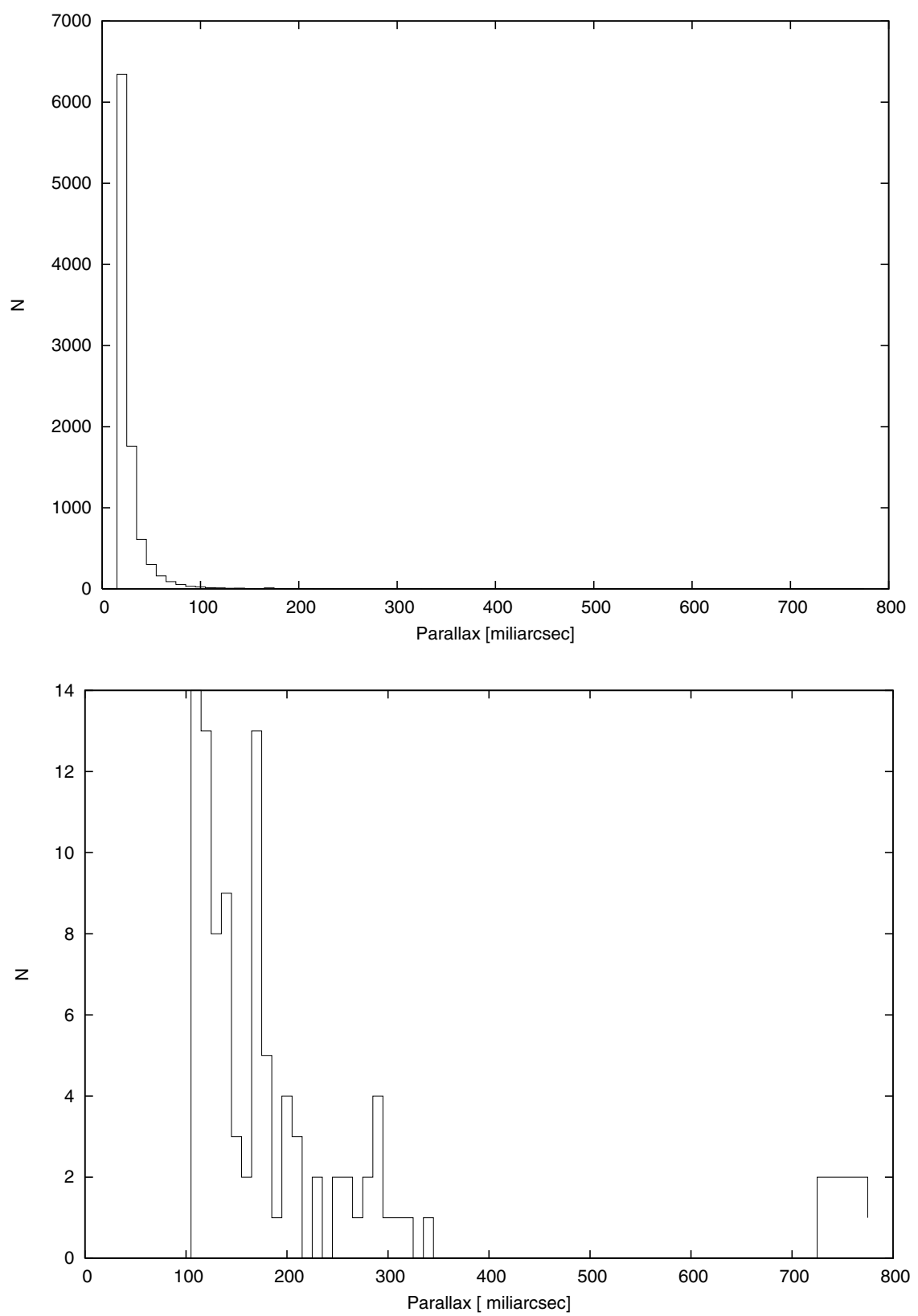

Figure 2. The parallax distribution

Above - Sample 1, Below - Sample 2.

deviation, the difference is not so prominent. This fact is interpreted by us as due to the observational selection. The best way to demonstrate its influence is to present the dependence mass-parallax (Fig. 4).

As clearly seen, the low-mass stars prevail at high parallaxes and vice versa. It is well known that high-mass stars are less frequent than low-mass ones. Therefore, within a small volume like that corresponding to a lower parallax limit of 0 ". 1 the probability of finding massive stars is low. On the other hand low-mass stars as less luminous (we are 

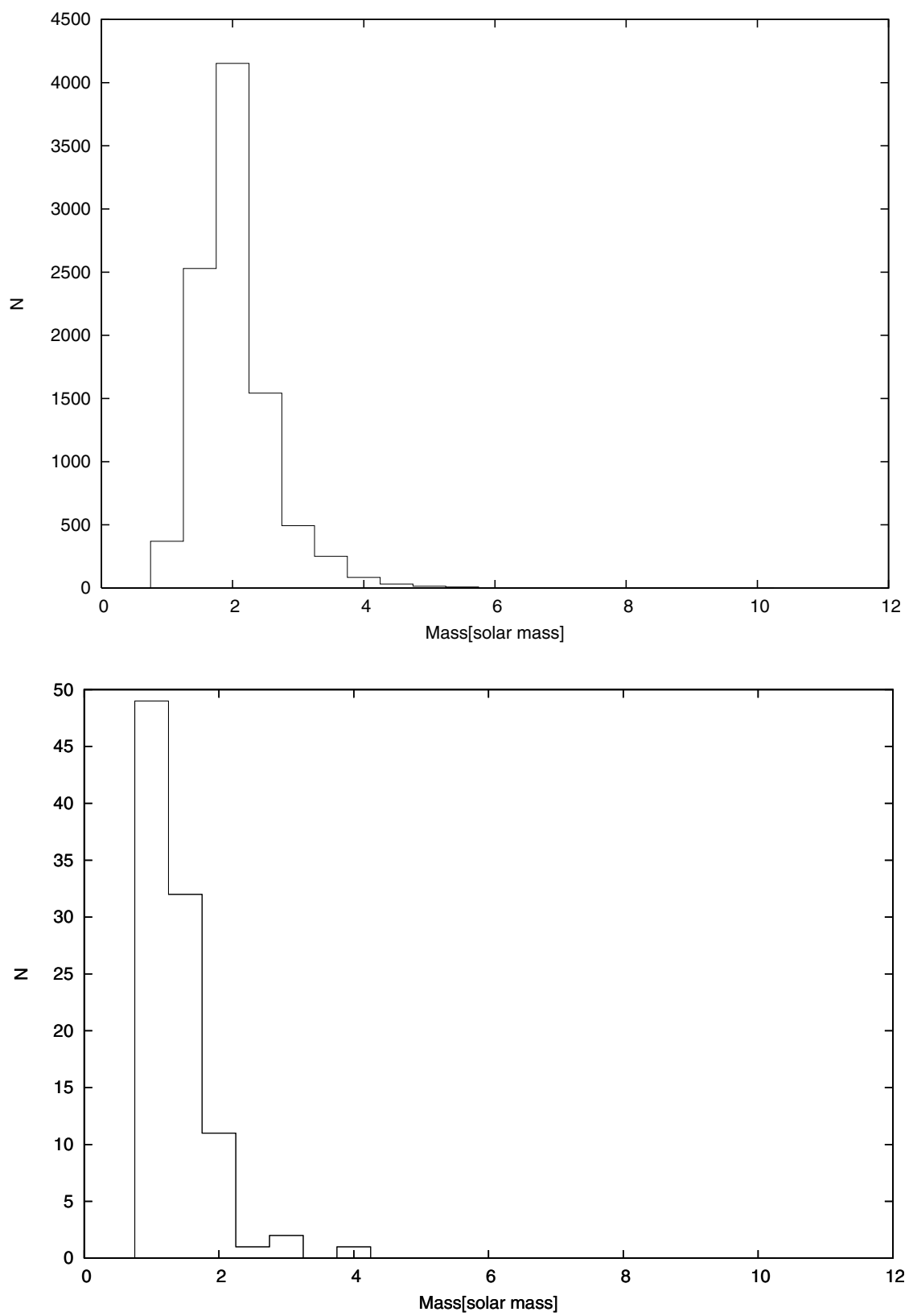

Figure 3. The mass distribution

Above - Sample 1, Below - Sample 2.

on MS!) are difficult to be discovered. The same is true for Agekyan's factor where the lack of low-mass stars produces an artificial equalling of star masses, i.e. diminishes the mass scatter. Nevertheless, it is even for very close stars still significantly smaller than the value expected on the basis of high numbers (Ninković 1995). As a possible explanation we offer the influence of the accuracy with which the parallaxes used here are obtained. The estimates of the number density of stars and their mean mass performed at the same 


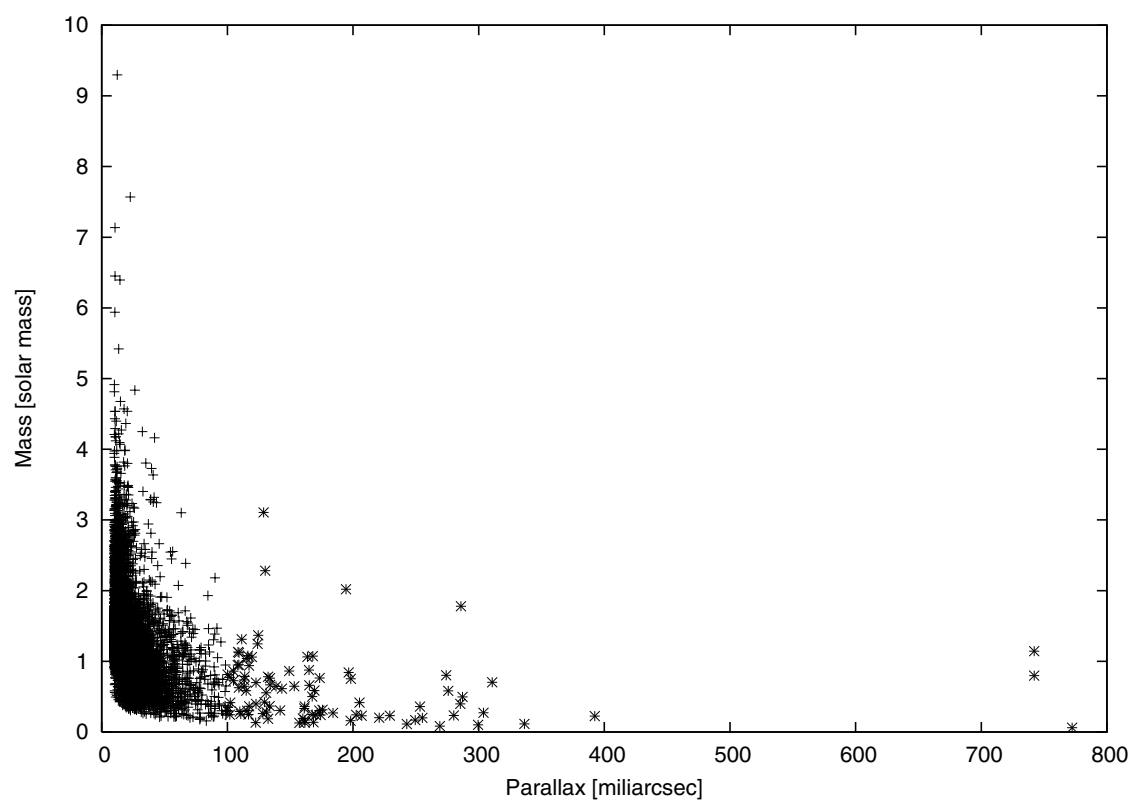

Figure 4. Dependence Mass-Parallax for Stars of Sample 1.

location are correlated; this indicates problems with the fainter end of MS, irrespectively of the precision of our results.

\section{Acknowledgements}

This work is a part of Project 1468 supported by the Ministry for Science and Environmental Protection of the Republic of Serbia.

\section{References}

Agekyan, T.A. 1962, in Kurs astrofiziki i zvezdnoj astronomii, tom vtoroj, ed. A. A. Mikhajlov, Gos. izd. fiz. - mat. lit., Moskva, p. 528

Angelov, T. 1993, Bull. Astron. Belgrade 148, 1

Binney, J. \& Tremaine, S. 1987, Galactic Dynamics, "Princeton University Press", Princeton, New Jersey

ESA (The Hipparcos Science Team: U. Bastian, P.L. Bernacca, M. Creze, F. Donati, M. Grenon, M. Grewing, E. Hg (TDAC Consortium Leader), J. Kovalevsky, (FAST Consortium Leader), F. van Leeuwen, L. Lindegren, (NDAC Consortium Leader), H. van der Marel, F. Mignard, C.A. Murray, M.A.C. Perryman, ESA (Chairman) Hipparcos Project Scientist, R.S. Le Poole, H. Schrijver, C. Turon (INCA Consortium Leader)) 1997, The Hipparcos and Tycho Catalogues ESA SP - 1200

Marochnik, L.S. \& Suchkov, A.A. 1984, Galaktika, "Nauka", glav. red. fiz. - mat. lit., Moskva Ninković, S. 1995, Bull. Astron. Belgrade 151, 1

Trajkovska, V. \& Ninković, S. 1997, Bull. Astron. Belgrade 155, 35 